\title{
A Study on the Errors in the Free-Gyro Positioning and Directional System
}

\author{
+ Tae-Gweon Jeong
}

+ Division of Navigation Science, Korea Maritime University, Pusan 606-791, Republic. of Korea

\begin{abstract}
This paper is to develop the position error equations including the attitude errors, the errors of nadir and ship's heading, and the errors of ship's position in the free-gyro positioning and directional system. In doing so, the determination of ship's position by two free gyro vectors was discussed and the algorithmic design of the free-gyro positioning and directional system was introduced briefly. Next, the errors of transformation matrices of the gyro and body frames, i.e. attitude errors, were examined and the attitude equations were also derived. The perturbations of the errors of the nadir angle including ship's heading were investigated in each stage from the sensor of rate of motion of the spin axis to the nadir angle obtained. Finally, the perturbation error equations of ship's position used the nadir angles were derived in the form of a linear error model and the concept of FDOP was also suggested by using covariance of position error.
\end{abstract}

Key words : free-gyro, attitude error, position error equation, FDOP, nadir angle, linear error model

\section{Introduction}

A free-gyro positioning system is to determine the position of a vehicle by using two free gyros. It is an active positioning system like an inertial navigation system (INS) in view of obtaining a position without external source. However, the FPS is to determine its own position by using the nadir angle between the vertical axis of local geodetic frame and the axis of free gyro, while an INS is to do it by measuring its acceleration.

In general the INS comprises a set of inertial measurement units (IMU's), both accelerometers and gyros, the platform on which they are mounted, including the stabilization mechanism if so provided, and the computer that performs the calculations needed to transform sensed accelerations and, in some mechanizations, angles or angular rates into navigationally useful information such as position, velocity and attitude. Therefore it is a very complicated structure.

On the other hand, the free-gyro positioning and directional system consists of a set of two sensors of gyro axis motion rate and three sensors of the body frame, two free gyros, and the computer that calculates navigational information, position, etc. It is comparatively simpler than the INS
Park \& Jeong (2004) investigated how to determine the gyro vectors of two free gyros and the position of a vehicle by using the gyros. The errors in the FPS were investigated broadly by Jeong (2005). And the algorithmic design of free gyroscopic compass and positioning was suggested by measuring the earth's rotation rate on the basis of a free gyroscope (Jeong \& Park, 2006; Jeong \& Park, 2011)

Meanwhile, the free-gyro positioning and directional system is thought to have its own errors. This paper is to analyze such errors theoretically. Firstly, the errors of transformation matrices of the gyro and body frames, i.e. attitude errors, will be examined and the attitude equations be also derived. The perturbations of the errors of the nadir angle including ship's heading will be investigated in each stage from the sensor of rate of motion of the spin axis to the nadir angle obtained. Finally, the perturbation error equations of ship's position used the nadir angles will be derived in the form of a linear error model and the concept of FDOP will be also suggested by using covariance of position error.

Before the errors involved are discussed, the overview of the free-gyro positioning and directional system will be presented

\footnotetext{
† Corresponding author, tgjeong@hhu.ac.kr 051)410-4246
} 


\section{Overview of free-gyro positioning and directional system}

\subsection{Determination of ship's position}

The nadir angle, $\theta$, is given by an arbitrary position and gyro vector as shown as equation (1).

$$
\begin{aligned}
\cos \theta= & -u_{x} \cos \phi \cos \left(\lambda+\omega_{e} t\right) \\
& -u_{y} \cos \phi \sin \left(\lambda+\omega_{e} t\right)-u_{z} \sin \phi
\end{aligned}
$$

Here, $\omega_{e}$ is the (presumably uniform) rate of Earth rotation, $\lambda$ the geodetic longitude, $\phi$ the geodetic latitude and $t$ the time. And $u_{x}, u_{y}$ and $u_{z}$ are the components of the gyro vector, $g^{i}$, whose superscript indicates the inertial frame. If we use two gyro vectors of $g_{a}^{i}=\left[u_{a x}, u_{a y}, u_{a z}\right]$ and $g_{b}^{i}=\left[u_{b x}, u_{b y}, u_{b z}\right]$ in (1), we can determine the position $(\phi, \lambda)$ of a vehicle by using two corresponding nadir angles $\theta_{a}$ and $\theta_{b}$.

Meanwhile the azimuth of the gyro vector from the north, $\alpha$, is represented by equation (2):

$\tan \alpha=\frac{E_{D}}{N_{D}}$,

where,

$$
\begin{aligned}
N_{D}= & -u_{x} \sin \phi \cos \left(\lambda+\omega_{e} t\right) \\
& -u_{y} \sin \phi \sin \left(\lambda+\omega_{e} t\right)+u_{z} \cos \phi \\
E_{D}= & -u_{x} \sin \left(\lambda+\omega_{e} t\right)+u_{y} \cos \left(\lambda+\omega_{e} t\right) .
\end{aligned}
$$

Once determining the position, we can also obtain the azimuth of a gyro vector by using Eq. (2). Park and Jeong (2004) already suggested the algorithm of how to determine a position.

Fig.1 shows the measurement quantities in the local navigation frame.

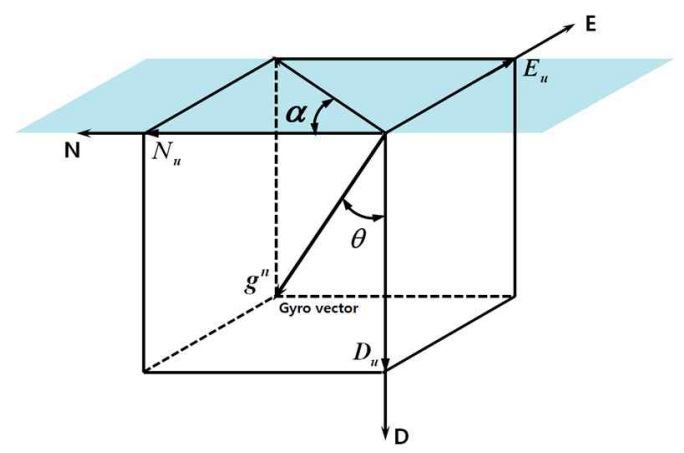

Fig. 1 Measurement quantities in the local navigation frame

\subsection{Relation between ship's heading and azimuth of gyro vector}

As Jeong \& Park (2006) mentioned, the north component of the earth's rotation rate is $\omega_{e} \cos \phi$. Fig. 2 shows that the angular velocities of the fore-aft and the athwartship components are given by equation (3) (Titterson, et al., 2004), where $\psi$ is ship's heading. And it also shows that $\zeta$ is the azimuth of a gyro vector from ship's head.

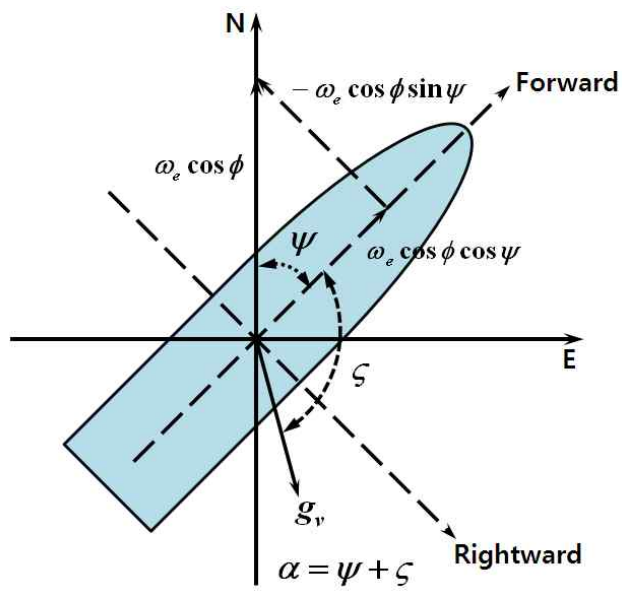

Fig. 2 Relation between ship's heading andazimuth of a gyro vector

$\omega_{N x}=\omega_{e} \cos \phi \cos \psi$

$\omega_{N y}=-\omega_{e} \cos \phi \sin \psi$

By taking the ratio of the two independent gyroscopic measurement, the heading,, is computed by (4).

$\psi=\tan ^{-1} \frac{\omega_{N y}}{\omega_{N x}}$

Meanwhile assuming that a gyro vector is $\zeta$ away from ship's head, its azimuth from North is represented by Eq. (5). Therefore the angular velocity of the horizontal axis of a gyro, $\omega_{H}$, is given by equation (6) on the navigation frame or local geodetic frame.

$\alpha=\psi+\zeta$

$\omega_{H}=-\omega_{e} \cos \phi \sin \alpha$

Equation (6) shows that if the north component of the earth's rotation rate can be known on the navigation frame, the nadir angle of a gyro vector, $\theta$, is obtained by (7), by integrating Eq. (6) incrementally over a time interval.

$\theta=\int_{t_{1}}^{t_{2}} \omega_{H} d t$ 


\subsection{Algorithmic design of free-gyro positioning and directional system}

Fig. 3 and Fig. 4 show the algorithmic design of free gyros positioning system mechanization. In this mechanization two sensors for sensing the motion rate of the spin axis are mounted in the free gyro. Three sensors for sensing the motion rate of the platform are mounted in orthogonal triad. From the sensors in the gyro frame, the spin motion rate, $\omega_{i / g}^{g}$, is obtained and from the ones in the body frame, $\omega_{i / b}^{b}$, is also detected. By using the sum, $\omega_{b / g}^{g}$, of the rates from the free gyro and the ones detected from the body sensors, the transformation matrix, $C_{g}^{b}$, and its inverse are determined. Therefore the spin motion rate, $\omega_{i / g}^{g}$, sensed from the free gyro is transformed into $\omega_{i / g}^{b}$ by using the matrix, $C_{g}^{b}$.

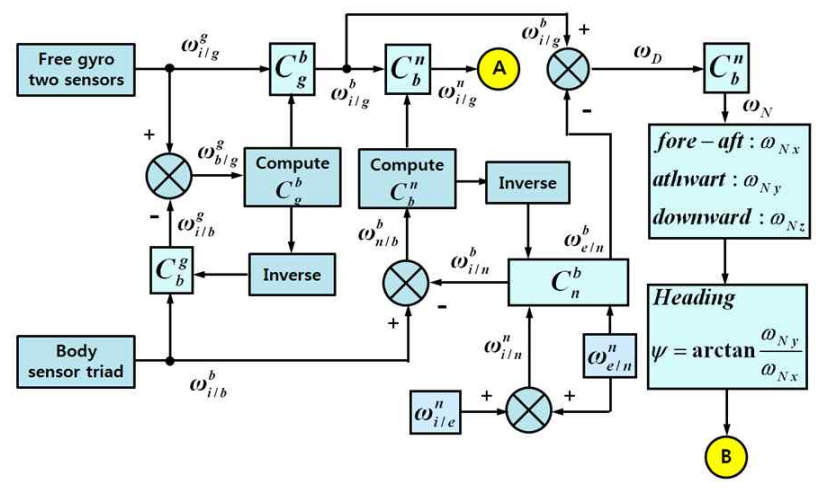

Fig. 3 Free gyro positioning and directional system mechanization (1)

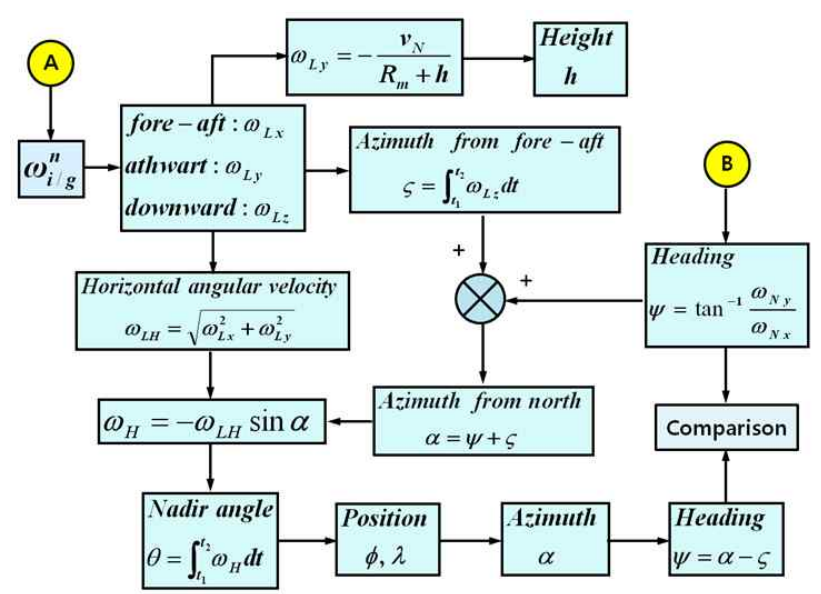

Fig. 4 Free gyro positioning and directional system mechanization (2)
Meanwhile the rate of the earth's rotation, $\omega_{i / e}^{n}$, and the rate of the vehicle movement, $\omega_{e / n}^{n}$, are added to make $\omega_{i / n}^{n}$. Here $\omega_{e / n}^{n}$ is given by:

$\omega_{e / n}^{n}=[\dot{\lambda} \cos \phi,-\dot{\phi},-\dot{\lambda} \sin \phi]^{T}$,

and $\omega_{i / n}^{n}$ is also expressed as:

$\omega_{i / n}^{n}=\left[\left(\dot{\lambda}+\omega_{e}\right) \cos \phi,-\dot{\phi},-\left(\dot{\lambda}+\omega_{e}\right) \sin \phi\right]^{T}$.

By using the matrix, $C_{n}^{b}$, it will be transformed into $\omega_{i / n}^{b}$, which is subtracted from the sensed rate from the body, $\omega_{i / b}^{b}$. As a result, $\omega_{n / b}^{b}$ is generated. By using this, the transformation matrix, $C_{b}^{n}$, and the inverse of it, $C_{n}^{b}$, are obtained. And the rate of $\omega_{i / g}^{b}$, is transformed into $\omega_{i / g}^{n}$ by using the matrix of $C_{b}^{n}$.

For ship's heading, by using the matrix of $C_{n}^{b}, \omega_{e / n}^{n}$ is changed into $\omega_{e / n}^{b}$, which is subtracted from $\omega_{i / g}^{b}$, and $\omega_{D}$ is calculated. Finally, using the transformation matrix, $C_{b}^{n}$, we can get the spin motion rate in the NED frame, $\omega_{N}$, where $\omega_{N}=\left[\omega_{N x}, \omega_{N y}, \omega_{N z}\right]^{T}$. As a result, the ship's heading, $\psi$, is calculated by using the components of the spin motion rate according to equation (4).

Next for ship's position, let's look into the nadir angle (Fig. 4). The motion rate of the spin axis in the navigation frame, $\omega_{i / g}^{n}$ is given by $\omega_{L}=\left[\omega_{L x}, \omega_{L y}, \omega_{L z}\right]^{T}$. The azimuth of the gyro vector from the ship's head, $\zeta$, can be obtained by integrating $\omega_{L z}$. Then the azimuth of the gyro vector from the North, $\alpha$, is obtained.

The horizontal angular velocity, $\omega_{L H}$, and the tilting rate, $\omega_{H}$, of the spin axis are calculated. And the nadir angle of the gyro vector, $\theta$, can be obtained by integrating $\omega_{H}$. Finally we can get the ship's position expressed by $(\phi, \lambda)$, using equation (1).

Next, using equation (2), we can also obtain the azimuth of the gyro vector, $\alpha$, and ship's heading, $\psi$, which are modified by iteration. And ship's position is also corrected. In addition if only we know the northward component of ship's speed, we can also obtain the height, $h$. 
A Study on the Errors in the Free-Gyro Positioning and Directional System

\section{PERTURBATION FORM OF ERROR EQUATIONS}

This paper derives the error equations of the position and attitude by using linear error model forms (Jekeli, 2001; Roger, 2007).

\subsection{Gyro frame and body frame error equations}

Gyro frame error equations can be derived by the transformation matrix, $C_{g}^{b}$. The estimated or computed matrix of it is represented by (8):

$\overline{C_{g}^{b}}=(I-\Xi) C_{g}^{b}$,

where $\Xi$ is a skew-symmetric matrix, which is equivalent to the vector, $\xi=\left[\xi_{x}, \xi_{y}, \xi_{z}\right]^{T}$, and is given by:

$$
\Xi=\left[\begin{array}{ccc}
0 & -\xi_{z} & \xi_{y} \\
\xi_{z} & 0 & -\xi_{x} \\
-\xi_{y} & \xi_{x} & 0
\end{array}\right]
$$

The differentiation form of $C_{g}^{b}$ can be represented by the following

$$
\dot{C}_{g}^{b}=C_{g}^{b} \Omega_{b / g}^{g}
$$

The error matrix of $C_{g}^{b}$ is also given by:

$$
\begin{aligned}
\delta C_{g}^{b} & =\overline{C_{g}^{b}}-C_{g}^{b} . \\
& =-\Xi C_{g}^{b}
\end{aligned}
$$

Taking the derivative of (10) yields

$$
\begin{aligned}
\delta C_{g}^{b} & =-\dot{\Xi} C_{g}^{b}-\Xi \dot{C}_{g}^{b} \\
& =-\dot{\Xi} C_{g}^{b}-\Xi C_{g}^{b} \Omega_{b / g}^{g}
\end{aligned}
$$

The error equation of equation (9) is given by:

$$
\begin{aligned}
\delta \dot{C}_{g}^{b} & =\delta\left(C_{g}^{b} \Omega_{b / g}^{g}\right) \\
& =\delta C_{g}^{b} \Omega_{b / g}^{g}+C_{g}^{b} \delta \Omega_{b / g}^{g}
\end{aligned}
$$

where the perturbation in angular rate, $\delta \Omega_{b / g}^{g}$, denotes the error in the computed value, $\delta \overline{\Omega_{b / g}^{g}}$, and is expressed as:

$\delta \Omega_{g}^{b}=\overline{\Omega_{b / g}^{b}}-\Omega_{b / g}^{b}$.
Substituting (13) into (12) and equating (12) with (11), we can get

$-\dot{\Xi} C_{g}^{b}-\Xi C_{g}^{b} \Omega_{b / g}^{g}=\delta C_{g}^{b} \Omega_{b / g}^{g}+C_{g}^{b} \delta \Omega_{b / g}^{g}$.

Substituting (10) into (14) and arranging it yields:

$-\dot{\Xi}=-C_{g}^{b} \delta \Omega_{b / g}^{g} C_{b}^{g}$

This is equivalent to the vector form given by:

$\dot{\xi}=-C_{g}^{b} \delta \omega_{b / g}^{g}$

where $\delta \omega_{b / g}^{g}$ is the error in the rotation rate of the gyro frame relative to the body frame. This is separated by:

$\omega_{b / g}^{g}=\omega_{i / g}^{g}-C_{b}^{g} \omega_{i / b}^{b}$

An error of this equation will be represented by:

$\delta \omega_{b / g}^{g}=\delta \omega_{i / g}^{g}-\left(C_{b}^{g} \Xi \omega_{i / b}^{b}+C_{b}^{g} \delta \omega_{i / b}^{b}\right)$.

Substituting (18) into (16) and rearranging it gives

$\dot{\xi}=-\omega_{i / b}^{b} \times \xi-C_{g}^{b} \delta \omega_{i / g}^{g}+\delta \omega_{i / b}^{b}$.

Equation (19) shows the error dynamics of the gyro frame attitude.

Similarly, we can get the error equations of the body frame attitude as the following (20).

$\dot{\gamma}=-\omega_{i / n}^{n} \times \gamma-C_{b}^{n} \delta \omega_{i / b}^{b}+\delta \omega_{i / n}^{n}$

Here $\gamma$ is the error angle of body frame attitude given by $\gamma=\left[\gamma_{x}, \gamma_{y}, \gamma_{z}\right]^{T}$. This can be represented equivalently by the skew-symmetric matrix, $\Gamma$.

$\Gamma=\left[\begin{array}{ccc}0 & -\gamma_{z} & \gamma_{y} \\ \gamma_{z} & 0 & -\gamma_{x} \\ -\gamma_{y} & \gamma_{x} & 0\end{array}\right]$

In addition, the error of the rate of motion of the navigation frame, $\delta \omega_{i / n}^{n}$, is expressed as:

$\delta \omega_{i / n}^{n}=\left[\begin{array}{c}\delta \dot{\lambda} \cos \phi-\left(\dot{\lambda}+\omega_{e}\right) \delta \phi \sin \phi \\ -\delta \phi \\ \delta \dot{\lambda} \sin \phi-\left(\dot{\lambda}+\omega_{e}\right) \delta \phi \cos \phi\end{array}\right]$ 


\section{Tae-Gweon Jeong}

3.2 Perturbations of error equations for nadir angle and ship's heading

In Fig. (3), the rate of motion of the spin axis in the navigation frame, $\omega_{i / g}^{n}$ is given by;

$\omega_{i / g}^{n}=C_{b}^{n} C_{g}^{b} \omega_{i / g}^{g}$.

We can get the error of this equation by using the differential operator, $\delta$.

$\delta \omega_{i / g}^{n}=-\left(\Gamma C_{b}^{n}+C_{b}^{n} \Xi\right) C_{g}^{b} \omega_{i / g}^{g}+C_{b}^{n} C_{g}^{b} \delta \omega_{i / g}^{g}$

Here $\delta \omega_{i / g}^{n}=\left[\delta \omega_{L x}, \delta \omega_{L y}, \delta \omega_{L z}\right]^{T}$.

The error of the azimuth of the gyro vector from ship's head, $\delta \zeta$, and that of the north, $\delta \alpha$, are represented by:

$\delta \zeta=\int_{t_{1}}^{t_{2}} \delta \omega_{L z} d t$

$\delta \alpha=\delta \zeta+\delta \psi$.

The error of the horizontal rate of the spin axis, $\delta \omega_{L H}$, is expressed as:

$\delta \omega_{L H}=\frac{\omega_{L x} \delta \omega_{L x}+\omega_{L y} \delta \omega_{L y}}{\sqrt{\omega_{L x}^{2}+\omega_{L y}^{2}}}$.

And also the error of the tilting rate of the spin axis, $\delta \omega_{H}$, is obtained as:

$\delta \omega_{H}=-\delta \omega_{L H} \sin \alpha-\omega_{L H} \cos \alpha \delta \alpha$.

Finally, the error of the nadir angle of the gyro vector, $\delta \theta$, is given by:

$\delta \theta=\int_{t_{1}}^{t_{2}} \delta \omega_{H} d t$

Meanwhile the error of ship's heading is obtained as the following

The error of the rate of the motion of the spin axis in the body frame, $\delta \omega_{D}$, is expressed as:

$$
\begin{array}{r}
\delta \omega_{D}=-\Xi C_{g}^{b} \omega_{i / g}^{g}+C_{g}^{b} \delta \omega_{i / g}^{g} \\
-C_{n}^{b}\left(\Gamma \omega_{e / n}^{n}+\delta \omega_{e / n}^{n}\right)
\end{array}
$$

And the error of the rate of the motion of the spin axis in the navigation frame, $\delta \omega_{N}$, is given by:

$$
\begin{aligned}
\delta \omega_{N}= & -\Gamma C_{b}^{n} \omega_{D}-\left(\Gamma \omega_{e / n}^{n}+\delta \omega_{e / n}^{n}\right), \\
& +C_{b}^{n}\left(C_{g}^{b} \delta \omega_{i / g}^{g}-\Xi C_{g}^{b} \omega_{i / g}^{g}\right)
\end{aligned}
$$

where $\delta \omega_{N}=\left[\delta \omega_{N x} \delta \omega_{N y} \delta \omega_{N z}\right]$. Therefore the error of ship's heading, $\delta \psi$, is represented by:

$\delta \psi=\frac{\omega_{N x} \delta \omega_{N y}-\omega_{N y} \delta \omega_{N x}}{\omega_{N x}^{2} \sec ^{2} \psi}$

Fig.5 shows the error dynamics in the navigation frame we discussed so far.

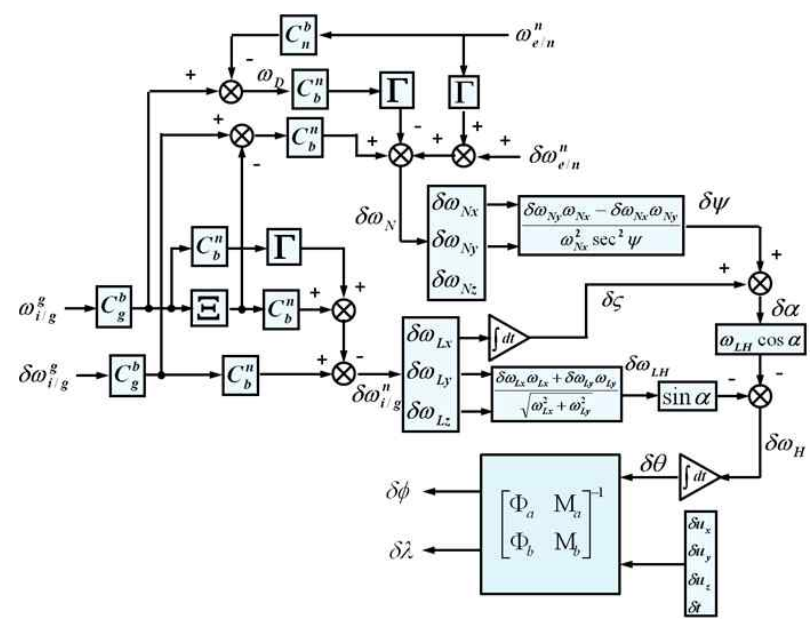

Fig. 5 Error dynamics of free gyro positioning and directional system

\subsection{Error equations of ship's position}

For a development of the position error equation, we differentially perturb eqn. (1), assuming that the earth rate is a constant, and then derive the perturbations of errors resulting in a linear model. The differential perturbation of eqn. (1) is given by eqn. (30):

$$
\begin{aligned}
& \cos \phi \cos \left(\lambda+\omega_{e} t\right) \delta u_{x}+\cos \phi \sin \left(\lambda+\omega_{e} t\right) \delta u_{y} \\
& \quad+\sin \phi \delta u_{z}-\sin \theta \delta \theta \\
& \quad+\cos \phi\left[u_{y} \cos \left(\lambda+\omega_{e} t\right)+u_{x} \sin \left(\lambda+\omega_{e} t\right)\right] \omega_{e} \delta t \\
& =\left[u_{x} \sin \phi \cos \left(\lambda+\omega_{e} t\right)+u_{y} \sin \phi \sin \left(\lambda+\omega_{e} t\right)-u_{z} \cos \phi\right] \delta \phi \\
& \quad+\left[u_{x} \cos \phi \sin \left(\lambda+\omega_{e} t\right)-u_{y} \cos \phi \cos \left(\lambda+\omega_{e} t\right)\right] \delta \lambda
\end{aligned}
$$

Now, let two gyro-vectors be $g_{a}^{i}=\left[u_{a x} u_{a y} u_{a z}\right]^{T}$ and $g_{b}^{i}=\left[\begin{array}{lll}u_{b x} & u_{b y} & u_{b z}\end{array}\right]^{T}$. And the corresponding nadir angles of 
A Study on the Errors in the Free-Gyro Positioning and Directional System

$\theta_{a}$ and $\theta_{b}$ are given. The perturbation matrix is arranged by eqn. (31).

$$
\left[\begin{array}{c}
Z_{a} \\
Z_{b}
\end{array}\right]=\left[\begin{array}{ll}
\Phi_{a} & M_{a} \\
\Phi_{b} & M_{b}
\end{array}\right]\left[\begin{array}{l}
\delta \phi \\
\delta \lambda
\end{array}\right]
$$

Here,

$$
\begin{aligned}
Z_{a}= & \cos \phi \cos \left(\lambda+\omega_{e} t\right) \delta u_{a x}+\cos \phi \sin \left(\lambda+\omega_{e} t\right) \delta u_{a y}, \\
& +\sin \phi \delta u_{a z}-\sin \theta_{a} \delta \theta_{a} \\
& +\cos \phi\left[u_{a y} \cos \left(\lambda+\omega_{e} t\right)-u_{a x} \sin \left(\lambda+\omega_{e} t\right)\right] \omega_{e} \delta t \\
Z_{b}= & \cos \phi \cos \left(\lambda+\omega_{e} t\right) \delta u_{b x}+\cos \phi \sin \left(\lambda+\omega_{e} t\right) \delta u_{b y}, \\
& +\sin \phi \delta u_{b z}-\sin \theta_{b} \delta \theta_{b} \\
& +\cos \phi\left[u_{b y} \cos \left(\lambda+\omega_{e} t\right)-u_{b x} \sin \left(\lambda+\omega_{e} t\right)\right] \omega_{e} \delta t \\
\Phi_{a}= & u_{a x} \sin \phi \cos \left(\lambda+\omega_{e} t\right) \\
& +u_{a y} \sin \phi \sin \left(\lambda+\omega_{e} t\right)-u_{a z} \cos \phi \\
\Phi_{b}= & u_{b x} \sin \phi \cos \left(\lambda+\omega_{e} t\right) \\
& +u_{b y} \sin \phi \sin \left(\lambda+\omega_{e} t\right)-u_{b z} \cos \phi \\
M_{a}= & u_{a x} \cos \phi \sin \left(\lambda+\omega_{e} t\right)-u_{a y} \cos \phi \cos \left(\lambda+\omega_{e} t\right), \\
M_{b}= & u_{b x} \cos \phi \sin \left(\lambda+\omega_{e} t\right)-u_{b y} \cos \phi \cos \left(\lambda+\omega_{e} t\right)
\end{aligned}
$$

This matrix can be written in the matrix-vector form,

$\triangle Z=\Psi \triangle x \Leftrightarrow \triangle x=\Psi^{-1} \triangle Z$,

where,

$\Delta x=\left[\begin{array}{c}\delta \phi \\ \delta \lambda\end{array}\right], \Psi^{-1}=\left[\begin{array}{ll}\Phi_{a} & M_{a} \\ \Phi_{b} & M_{b}\end{array}\right]^{-1}$, and $\Delta Z=\left[\begin{array}{c}Z_{a} \\ Z_{b}\end{array}\right]$

have been introduced. The covariance matrix for eqn. (32) is given by:

$\operatorname{cov}(\triangle x)=\Psi^{-1} \operatorname{cov}(\triangle Z)\left(\Psi^{-1}\right)^{T}$.

The assumption may be made that the sensor and time errors show a random behavior resulting in a normal distribution with expectation value zero and variance, $\sigma_{s}^{2}$. Therefore, measured sensor and time values are linearly independent or uncorrelated. The $\operatorname{cov}(\Delta Z)$ is represented by:

$\operatorname{cov}(\triangle Z)=\sigma_{s}^{2} I$

where $I$ is the unit matrix.

Substituting eqn. (34) into eqn. (33) yields

$$
\begin{aligned}
\operatorname{cov}(\triangle x) & =\Psi^{-1} \sigma_{s}^{2} I\left(\Psi^{-1}\right)^{T}=\sigma_{s}^{2} \Psi^{-1}\left(\Psi^{-1}\right)^{T} \\
& =\sigma_{s}^{2}\left(\Psi^{T} \Psi\right)^{-1}=\sigma_{s}^{2} Q_{x}
\end{aligned}
$$

where $Q_{x}=\left(\Psi^{T} \Psi\right)^{-1}$.

The cofactor matrix, $Q_{x}$ (Hofmann-Wellenhof B. et al, 2001) is a 2 × 2 matrix where two components are contributed by the gyro vectors of $g_{a}^{i}$ and $g_{b}^{i}$. The elements of the cofactor matrix are denoted as:

$Q_{x}=\left[\begin{array}{ll}q_{\phi \phi} & q_{\phi \lambda} \\ q_{\phi \lambda} & q_{\lambda \lambda}\end{array}\right]$

In the cofactor matrix the diagonal elements are used for FDOP which is the geometry of two free gyros.

$F D O P=\sqrt{q_{\phi \phi}^{2}+q_{\lambda \lambda}^{2}}$

Therefore the error of a position in the free-gyro positioning and directional system, $f d_{\mathrm{s}}$, is given by:

$f d_{\mathrm{s}}=F D O P \times \sigma_{s}$

From eqn. (38) the $f d_{s}$ can be computed easily. If in this case two-dimensional error distribution is close to being circular, the probability is about 0.63(Kaplan , 2006).

\section{Conclusions}

First of all, this paper dealt with the determination of ship's position by free gyros and its algorithmic design briefly. Next, the errors of transformation matrices of the gyro and body frames, i.e. attitude errors, were examined and the attitude equations were also derived. The perturbations of the errors of the nadir angle including ship's heading were investigated in turn. Finally, the perturbation error equations of ship's position used the nadir angles were derived in the form of a linear error model and the concept of FDOP was also suggested by using covariance of position error.

However, the free-gyro positioning and directional system has still many problems to be solved. First the error of a position is experimentally verified. Especially the sensor errors will have to be investigated. In addition, the additional drift needs to be investigated, which occurs when a free gyro is suppressed by the measures which prevent 
gimbals lock and tumbling. The alignment in the system also needs to be examined.

All these will be dealt with in the next papers.

\section{References}

[1] Hofmann-Wellenhof B., Lichtenegger H. and Collins J.(2001), GPS, Theory and Practice, 5th edition, SpringerWien NewYork, p. 272.

[2] Jekeli, C.(2001), Inertial Navigation Systems with Geodetic Applications, Walter de Gruyter, p. 147-157.

[3] Jeong, T. G.(2005), "A Study on the Errors in the Free-Gyro Positioning System ( I )”, International Journal of Navigation and Port Research, Vol. 29, No. 7, pp. 611-614.

[4] Jeong, T. G. and Park, S. C.(2006), "A Theoretical Study on Free Gyroscopic Compass", International Journal of Navigation and Port Research, Vol. 30, No. 9, pp. 729-734.

[5] Jeong, T. G. and Park, S. C.(2011), "An Algorithmic Study on Positioning and Directional System by Free Gyros”, Navigational Systems and Simulators, CRC Press, pp. 79-84.

[6] Kaplan, E. D. et al(2006), Understanding GPS Principles and Application, 2nd edition, ARTECH House, p. 331.

[7] Park, S.C. and Jeong, T.G.(2004), "A Basic Study on Position Fixing by Free Gyros", Journal of Korean Navigation and Port Research, Vol. 28, No. 8, pp. 653-657.

[8] Rogers, R.M(2007), Applied Mathematics in Integrated Navigation Systems, 3rd ed., AIAA, pp. 106-111.

[9] Titterton, D.H. and Weston, J.L.(2004), Strapdown Inertial Navigation Technology, 2nd ed., AIAA, p. 287.

Received 14 May 2013

Revised 12 June 2013

Accepted 12 June 2013 